\title{
Theoretical Comparison of Methods for Modeling Thin Dielectric or Conducting Sheets in the FDTD Grid
}

DOI:

10.1109/TAP.2018.2874754

\section{Document Version}

Accepted author manuscript

Link to publication record in Manchester Research Explorer

\section{Citation for published version (APA):}

Bérenger, J. P., \& Costen, F. (2018). Theoretical Comparison of Methods for Modeling Thin Dielectric or Conducting Sheets in the FDTD Grid. IEEE Transactions on Antennas and Propagation .

https://doi.org/10.1109/TAP.2018.2874754

\section{Published in:}

IEEE Transactions on Antennas and Propagation

\section{Citing this paper}

Please note that where the full-text provided on Manchester Research Explorer is the Author Accepted Manuscript or Proof version this may differ from the final Published version. If citing, it is advised that you check and use the publisher's definitive version.

\section{General rights}

Copyright and moral rights for the publications made accessible in the Research Explorer are retained by the authors and/or other copyright owners and it is a condition of accessing publications that users recognise and abide by the legal requirements associated with these rights.

\section{Takedown policy}

If you believe that this document breaches copyright please refer to the University of Manchester's Takedown Procedures [http://man.ac.uk/04Y6Bo] or contact uml.scholarlycommunications@manchester.ac.uk providing relevant details, so we can investigate your claim.

\section{OPEN ACCESS}




\title{
Theoretical Comparison of Methods for Modeling Thin Dielectric or Conducting Sheets in the FDTD Grid
}

\author{
Jean-Pierre Bérenger, Fellow, IEEE, Fumie Costen, Senior Member, IEEE
}

\begin{abstract}
This paper compares the methods used to account for sheets thinner than the cell size of the FDTD method. The comparison relies on the reflection and transmission of plane waves. It is shown that the Maloney and Smith method is the best. In contrast to the other methods, it provides correct reflection and transmission coefficients for any incidence angle.
\end{abstract}

Index Terms-FDTD methods, electromagnetic coupling, reflection coefficient.

\section{INTRODUCTION}

Several techniques were published almost simultaneously [1]-[6] to enable the finite-difference time-domain (FDTD) method [7]-[8] to deal with dielectric or conducting sheets thinner than the cell size. A comparison by means of numerical experiments [9] showed that using [1] the computed results agree with the analytical solution, while using [2]-[5] they experience some discrepancies when the electric field is perpendicular to the sheet.

In this paper we revisit the comparison of the techniques by means of theory. The comparison is limited to [1] and [3], since [4] and [6] are similar to [3], [2] is limited to dielectric sheets, and [5] is subject to instability. The theoretical reflection and transmission of an incident plane wave that strikes a thin sheet are derived in the FDTD discretized space. They are compared with the analytical reflection and transmission in the physical continuous space. This shows that using [3] the reflection and transmission are correct when the incidence is close to normal incidence, but erroneous at wide incidence. Conversely, with [1] they agree with their physical counterparts at all incidence angles. In particular, the zero reflection at the Brewster angle is well reproduced with a dielectric sheet.

\section{The Maloney-Smith And LuebBers-KunZ Techniques}

With techniques [3] or [6], the thin sheet can be assumed as centered on a plane that holds the two $E$ components parallel to the sheet, as represented in Fig. 1, where the nodes $E_{y}$ and $E_{z}$ are within the sheet. By discretizing the integral form of the Maxwell-Ampère equation, it is found that the FDTD advance of the two $E$ components in the sheet can be performed with the standard FDTD equation, with permittivity and conductivity replaced by their average value in the cell surrounding the considered node. Thus, for a sheet surrounded by air, of thickness $d$, relative permittivity $\varepsilon_{s}$, and conductivity $\sigma_{s}$, the relative permittivity and conductivity to be used in the sheet are

$$
\varepsilon_{r}=1-\frac{d}{\Delta x}+\varepsilon_{s} \frac{d}{\Delta x} \quad ; \quad \sigma=\sigma_{s} \frac{d}{\Delta x} .
$$

This method yields correct results in many experiments [3], [6]. However, it is evident that when a plane wave propagates in a direction parallel to the sheet (in the $y$ - $z$ plane in Fig. 1) with $E$ perpendicular to the sheet, there is no $E$ component parallel to the sheet, and thus this propagation in the FDTD space is just like the one in a vacuum, i.e. the sheet is transparent to the wave.

Manuscript received May 8, 2018.

J.-P. Bérenger and F. Costen are with the School of Electrical and Electronic Engineering, The University of Manchester, Manchester, M193PL, UK. (email: jpberenger@gmail.com; fumie.costen@manchester.ac.uk).

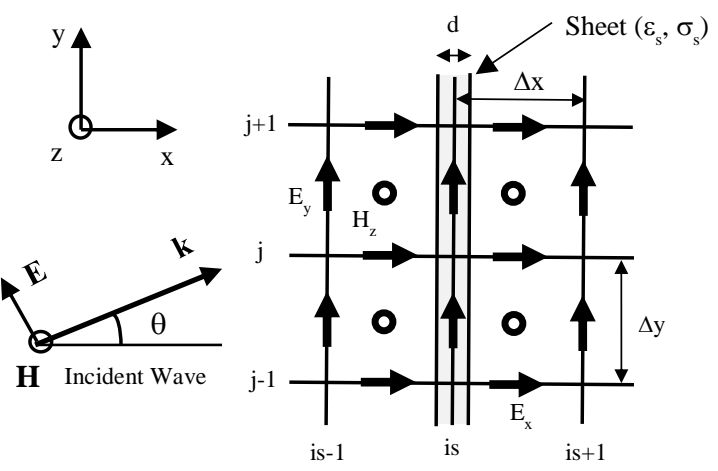

Fig. 1. A plane $x-y$ of a 3D FDTD mesh with a thin sheet located at $i=i s$.

We do not face this problem with the Maloney and Smith additional modification to the FDTD equations [1]. Field $E$ perpendicular to the sheet $\left(E_{x}\right.$ at $i=i s-1 / 2$ in Fig. 1) is split into two parts, one advanced as in a vacuum, the other advanced as if it were in the sheet. A weighted average of the two parts in then used in place of $E$ in the FDTD advance of $H$ components parallel to the sheet. In the case of Fig. 1, denoting the two parts as $E_{x_{-} \text {out }}$ and $E_{x_{-} i n}$, component $E_{x}$ is replaced with $\alpha E_{x_{-} \text {out }}$ $+\beta E_{x_{-} i n}$ in the advance of $H_{z}$ in the plane $i=i s-1 / 2$, with

$$
\alpha=1-d / \Delta x \quad \beta=d / \Delta x .
$$

Experiments in a special case in [9] showed a better agreement of analytical solutions with the Maloney and Smith method than with other methods. This is confirmed in the following sections by investigating the reflection and transmission of plane waves by thin sheets.

\section{Reflection and Transmission of Plane Waves}

In this section, we review the formula giving the physical reflection and transmission by a sheet, we derive the reflection and transmission in the FDTD grid, and we briefly describe how the reflection can be measured by FDTD experiments.

\section{A. Analytical reflection and transmission of a sheet}

It is a simple exercise to derive the reflection $R_{a}$ and transmission $T_{a}$ of a plane wave by a sheet of thickness $d$, relative permittivity $\varepsilon_{s}$ and conductivity $\sigma_{s}$. This yields

$$
R_{a}=r_{0}-\frac{r_{0}\left(1-r_{0}^{2}\right) \phi^{2}}{1-r_{0}^{2} \phi^{2}} \quad ; \quad T_{a}=\frac{\left(1-r_{0}^{2}\right) \phi}{1-r_{0}^{2} \phi^{2}}
$$

where, for incidence $\theta$ and with $\varepsilon_{r}=\varepsilon_{s}$-j $\sigma_{s} / \varepsilon_{0} \omega$ and $c=1 /\left(\varepsilon_{0} \mu_{0}\right)^{1 / 2}$

$$
r_{0}=\frac{\varepsilon_{r} \cos \theta-\sqrt{\varepsilon_{r}-\sin ^{2} \theta}}{\varepsilon_{r} \cos \theta+\sqrt{\varepsilon_{r}-\sin ^{2} \theta}} \quad ; \phi=e^{-j \frac{\omega d}{c}\left(\sqrt{\varepsilon_{r}-\sin ^{2} \theta}+\frac{\sin ^{2} \theta}{\operatorname{Re} a\left[\sqrt{\varepsilon_{r}-\sin ^{2} \theta}\right]}\right)} .
$$

\section{B. Theoretical reflection and transmission in the FDTD grid}

We derive the FDTD reflection and transmission in the 2D TE case, which is also the $3 \mathrm{D}$ case if the incident wave propagates in the $x-y$ 
plane. The problem is thus the one in Fig. 1. Let us assume that the relative permittivity and the conductivity are $\left(\varepsilon_{a}, \sigma_{a}\right)$ for $i=i s-1,\left(\varepsilon_{b}\right.$, $\left.\sigma_{b}\right)$ for $i=i s$, and either $(1,0)$ or $\left(\varepsilon_{m}, \sigma_{m}\right)$ for $i=i s-1 / 2$, where $\left(\varepsilon_{m}, \sigma_{m}\right)$ are used for the advance of the additional $E_{x \_ \text {in }}$ component in Maloney method. The FDTD equations in the three columns are [1]

$$
\begin{aligned}
& E_{y}^{n+1}(i s-1, j+1 / 2)=A_{a} E_{y}^{n}(i s-1, j+1 / 2)-\frac{B_{a} \Delta t}{\varepsilon_{0} \Delta x} \\
& {\left[H_{z}^{n+1 / 2}(i s-1 / 2, j+1 / 2)-H_{z}^{n+1 / 2}(i s-3 / 2, j+1 / 2)\right]} \\
& E_{x_{-} \text {out }}^{n+1}(i s-1 / 2, j)=E_{x_{-} \text {out }}^{n}(i s-1 / 2, j)+\frac{\Delta t}{\varepsilon_{0} \Delta y} \\
& \left\lfloor H_{z}^{n+1 / 2}(i s-1 / 2, j+1 / 2)-H_{z}^{n+1 / 2}(i s-1 / 2, j-1 / 2)\right\rfloor \\
& E_{x_{-} i n}^{n+1}(i s-1 / 2, j)=A_{m} E_{x_{-} i n}^{n}(i s-1 / 2, j)+\frac{B_{m} \Delta t}{\varepsilon_{0} \Delta y} \\
& \left\lfloor H_{z}^{n+1 / 2}(i s-1 / 2, j+1 / 2)-H_{z}^{n+1 / 2}(i s-1 / 2, j-1 / 2)\right\rfloor \\
& H_{z}^{n+1 / 2}(i s-1 / 2, j+1 / 2)=H_{z}^{n-1 / 2}(i s-1 / 2, j+1 / 2) \\
& +\frac{\alpha \Delta t}{\mu_{0} \Delta y}\left[E_{x_{-} \text {out }}^{n}(\text { is }-1 / 2, j+1)-E_{x_{-} \text {out }}^{n}(\text { is }-1 / 2, j)\right] \\
& +\frac{\beta \Delta t}{\mu_{0} \Delta y}\left[E_{x_{-} i n}^{n}(i s-1 / 2, j+1)-E_{x_{-} i n}^{n}(i s-1 / 2, j)\right] \\
& -\frac{\Delta t}{\mu_{0} \Delta x}\left[E_{y}^{n}(i s, j+1 / 2)-E_{y}^{n}(i s-1, j+1 / 2)\right] \\
& E_{y}^{n+1}(i s, j+1 / 2)=A_{b} E_{y}^{n}(i s, j+1 / 2)-\frac{B_{b} \Delta t}{\varepsilon_{0} \Delta x} \\
& \left\lfloor H_{z}^{n+1 / 2}(i s+1 / 2, j+1 / 2)-H_{z}^{n+1 / 2}(i s-1 / 2, j+1 / 2)\right\rfloor
\end{aligned}
$$

where $\quad A_{u}=e^{-\sigma_{u} \Delta t / \varepsilon_{0} \varepsilon_{u}} ; B_{u}=\varepsilon_{0}\left(1-A_{u}\right) / \sigma_{u} \Delta t$.

Let us consider an incident wave of magnitude $E_{0 i}$ and wavenumbers $\left(k_{x}, k_{y}\right)$ propagating in direction $\theta$ with respect to direction $x$ (Fig. 1). Denoting by $\left(E_{y i}, H_{z i}\right)$ and $\left(E_{y r}, H_{z r}\right)$ the incident and reflected fields, and $R E_{0 i}$ the magnitude of the reflected wave, the field for $i<i s-1$, can be written as

$$
\begin{aligned}
& E_{y i}+E_{y r}=E_{0 i} \cos \theta\left[e^{-j k_{x} x}+\operatorname{Re}^{j k_{x} x}\right] e^{j \omega t-j k_{y} y} \\
& H_{z i}+H_{z r}=E_{0 i} \sqrt{\frac{\varepsilon_{0}}{\mu_{0}}}\left[e^{-j k_{x} x}-\operatorname{Re}^{j k_{x} x}\right] e^{j \omega t-j k_{y} y}
\end{aligned}
$$

where $x=0$ at $i=i$ s. Let us then write components $H_{z}(\mathrm{is}-1 / 2, \mathrm{j}+1 / 2)$, $E_{x_{-} \text {out }}($ is- $1 / 2, \mathrm{j})$, and $E_{x_{-} i n}($ is- $1 / 2, \mathrm{j})$ as

$$
\begin{aligned}
& H_{z}(i s-1 / 2, j+1 / 2)=T_{z} E_{0 i} e^{j \omega t-j k_{y} y} \\
& E_{x_{-} \text {out }}(i s-1 / 2, j)=T_{x_{-} \text {out }} E_{0 i} e^{j \omega t-j k_{y} y} \\
& E_{x_{-} \text {in }}(i s-1 / 2, j)=T_{x_{-} \text {in }} E_{0 i} e^{j \omega t-j k_{y} y}
\end{aligned}
$$

where $T_{z}, T_{x_{-} o u t}, T_{x_{-} i n}$ are unknown parameters, and $k_{y}$ is the same as in (5), following the Snell law which holds in the discretized medium. In the vacuum, for $i>i s$, the transmitted wave can be written as

$$
\begin{gathered}
E_{y t}=T E_{0 i} \cos \theta e^{j \omega t-j k_{x} x-j k_{y} y} \\
H_{z t}=T \sqrt{\frac{\varepsilon_{0}}{\mu_{0}}} E_{0 i} e^{j \omega t-j k_{x} x-j k_{y} y}
\end{gathered}
$$

where again $k_{y}$ is the same as in (5) and thus the direction of propagation $\theta$ is also the same as in (5).

By inserting waves (5)-(7) into the FDTD equations (4), we obtain a set of five equations for the five unknown parameters $R, T_{z}, T_{x_{-} \text {out }}$, $T_{x \_i n}$, and $T$ as in

$$
\begin{aligned}
& 2 j \cos \theta(1+R) \Sigma_{a}=-\frac{B_{a} c \Delta t}{\Delta x}\left[T_{z}-e^{j k_{x} \Delta x / 2}+\mathrm{Re}^{-j k_{x} \Delta x / 2}\right] \\
& T_{x_{-} \text {out }} \sin (\omega \Delta t / 2)=-\frac{c \Delta t}{\Delta y} T_{z} \sin \left(k_{y} \Delta y / 2\right) \\
& T_{x_{-} \text {in }} \Sigma_{m}=-\frac{B_{m} c \Delta t}{\Delta y} T_{z} \sin \left(k_{y} \Delta y / 2\right) \\
& 2 j T_{z} \sin (\omega \Delta t / 2)= \\
& -2 j\left(\alpha T_{x_{-} \text {out }}+\beta T_{x_{-} \text {in }}\right) \frac{c \Delta t}{\Delta y} \sin \left(k_{y} \Delta y / 2\right)-\frac{c \Delta t}{\Delta x} \cos \theta(T-1-R) \\
& 2 j \cos \theta T \Sigma_{b}=-\frac{B_{b} c \Delta t}{\Delta x}\left[T e^{-j k_{x} \Delta x / 2}-T_{z}\right]
\end{aligned}
$$

where

$$
\Sigma_{u}=\frac{1}{2 j}\left[e^{j \omega \Delta t / 2}-A_{u} e^{-j \omega \Delta t / 2}\right]
$$

Solving the equations for reflection $R$ and transmission $T$ yields

$$
R=N / D \quad ; \quad T=-j B_{a} B_{b}\left(\alpha_{m}+\alpha_{p}\right) \chi / D
$$

where

$$
\begin{aligned}
& N=-\psi_{a} \psi_{b}+\left(\psi_{a} B_{b}+\psi_{b} B_{a}\right) \chi-B_{a} B_{b} \alpha_{m} \alpha_{p} \\
& -j\left\lfloor\psi_{b} B_{a} \alpha_{p}-\psi_{a} B_{b} \alpha_{m}-B_{a} B_{b}\left(\alpha_{p}-\alpha_{m}\right) \chi\right\rfloor \\
& D=\psi_{a} \psi_{b}-\left(\psi_{a} B_{b}+\psi_{b} B_{a}\right) \chi-B_{a} B_{b} \alpha_{m}^{2} \\
& -j\left[\alpha_{m}\left(\psi_{a} B_{b}+\psi_{b} B_{a}\right)-2 B_{a} B_{b} \alpha_{m} \chi\right] \\
& \psi_{u}=2 \cos \theta \Sigma_{u} ; \chi=\frac{c^{2} \Delta t^{2}}{2 w \Delta x^{2}} \cos \theta \\
& w=\sin (\omega \Delta t / 2)-\frac{c^{2} \Delta t^{2}}{\Delta y^{2}}\left[\frac{\alpha}{\sin (\omega \Delta t / 2)}+\frac{\beta B_{m}}{\Sigma_{m}}\right] \sin ^{2}\left(k_{y} \Delta y / 2\right) \\
& \alpha_{m}=\frac{c \Delta t}{\Delta x} e^{-j k \Delta x / 2} ; \alpha_{p}=\frac{c \Delta t}{\Delta x} e^{j k \Delta x / 2}
\end{aligned}
$$

An interesting closed form can be obtained for a dielectric sheet where $\sigma_{s}=0, A_{u}=1, B_{u}=1 / \varepsilon_{u}$. When the frequency of interest is far below the cutoff frequency of the FDTD mesh, we have $\omega \Delta t<<1, k_{x}$ $\Delta x \ll 1, \omega=c k, k_{x}=\omega / c \cos \theta$, and $k_{y}=\omega / c \sin \theta$. Under such conditions, it can be seen that

$$
\begin{gathered}
\psi_{u} \approx \omega \Delta t \cos \theta ; \quad \chi \approx \frac{c^{2} \Delta t}{\Delta x^{2}} \frac{\Omega}{\omega} ; \chi \psi_{u} \approx \frac{c^{2} \Delta t^{2}}{\Delta x^{2}} \Omega \cos \theta \\
\left(\alpha_{p}-\alpha_{m}\right) \chi \approx j \frac{c^{2} \Delta t^{2}}{\Delta x^{2}} \Omega \cos \theta ; \Omega=\frac{\cos \theta}{1-\left(\alpha+\beta / \varepsilon_{m}\right) \sin ^{2} \theta}
\end{gathered}
$$

from which, denoting $\psi_{a}=\psi_{b}$ as $\psi$, at low frequency $R$ tends to

$$
R_{\text {low }}=\frac{\left(\varepsilon_{a}+\varepsilon_{b}\right) \psi \chi-\alpha_{m} \alpha_{p}+j\left(\alpha_{p}-\alpha_{m}\right) \chi}{j 2 \alpha_{m} \chi}
$$

Assuming that $\Delta y=\Delta x$ and replacing $\psi \chi$ and $\left(\alpha_{p}-\alpha_{m}\right) \chi$ with (10), and since $\alpha_{m} \alpha_{p}=c^{2} \Delta t^{2} / \Delta x^{2}$ at low frequency, $R_{\text {low }}$ reduces to

$$
R_{\text {low }}=-\frac{j}{2}\left[\left(\varepsilon_{a}+\varepsilon_{b}-1\right) \cos ^{2} \theta-1+\left(\alpha+\frac{\beta}{\varepsilon_{m}}\right) \sin ^{2} \theta\right] \frac{1}{\cos \theta} \frac{\omega}{c} \Delta x
$$

In the case $\varepsilon_{a}=1, \varepsilon_{b}=1+\left(\varepsilon_{s}-1\right) d / \Delta x, \alpha=1$, and $\beta=0$, which corresponds to technique [3], the reflection (12) becomes

$$
R_{\text {Luebbers }}=-\frac{j}{2}\left(\varepsilon_{s}-1\right) \cos \theta \frac{\omega}{c} d
$$

With the Maloney technique [1] with which $\varepsilon_{a}=1, \varepsilon_{b}=1+\left(\varepsilon_{s}-1\right) d / \Delta x$, $\varepsilon_{m}=\varepsilon_{\mathrm{s}}, \alpha=1-d / \Delta x$ and $\beta=d / \Delta x$, (12) yields

$$
R_{\text {Maloney }}=-\frac{j}{2}\left[\left(\varepsilon_{s}-1 / \varepsilon_{s}\right) \cos ^{2} \theta-1+1 / \varepsilon_{s}\right] \frac{1}{\cos \theta} \frac{\omega}{c} d
$$

The bracket in (14) vanishes for $\cos ^{2} \theta=1 /\left(\varepsilon_{s}+1\right)$ or equivalently 


$$
\tan (\theta)=\sqrt{\varepsilon_{s}} .
$$

This is the well-known Brewster angle where the reflection of the $E$ field lying in the plane of incidence vanishes at the interface between a vacuum and a dielectric of relative permittivity $\varepsilon_{s}$. If the reflection from the two interfaces of a sheet is zero, the overall reflection should be zero. This is well verified with the Maloney technique, at least in the frequency ranges well below the mesh cutoff. In contrast, reflection (13) of [3] never vanishes. This clearly demonstrates the superiority of the Maloney technique, which is confirmed in the following.

\section{Calculation of the reflection by means of FDTD experiments}

The reflection coefficient from a boundary condition [10] can be measured within a large 2D FDTD domain where the incident wave is produced by a Huygens surface. The reader is referred to [10] for more details on the settings. The technique has been used here to measure the reflection from a thin sheet so as to validate the theoretical FDTD reflection (9).

\section{COMPARISON OF Methods}

In Figs 2-6, method [3] is denoted as «Luebbers », and method [1] is denoted as «Maloney ». They are compared in Figs 2 and 3 for a dielectric sheet of $\varepsilon_{s}=10$ and $d=1 \mathrm{~cm}$. The space step of the cubic FDTD cell is $5 \mathrm{~cm}$, so that $d=\Delta x / 5$, and the time step is $0.1 \mathrm{~ns}$. In Fig. 2 the incidence is $\theta=80^{\circ}$ and results are plotted as a function of frequency (the cutoff of the FDTD mesh is about $2 \mathrm{GHz}$ ). FDTD experiments, FDTD reflection (9), and analytical reflection (3), are plotted in Fig. 2. The theoretical formula (9) agrees very well with FDTD experiments with both Luebbers and Maloney methods. But the Maloney results are very close to the analytical solution whilst the Luebbers results differ by $7-8 \mathrm{~dB}$.

Fig. 3 provides with another view of the same case. Here Maloney and Luebbers reflections (9) are compared with the analytical reflection (3) as a function of the incidence $\theta$, at frequencies $10 \mathrm{MHz}$ and $100 \mathrm{MHz}$ (about 1/200 and 1/20 of the FDTD cutoff). Again, Maloney results agree very well with the analytical solution across the whole range of incidences. Luebbers results are correct up to incidence $45^{\circ}$, but at a wider incidence they deviate from the analytical solution. This was expected from (13) and (14). While Luebbers reflection is a monotonic function of $\theta$, Maloney reflection (14) vanishes at the Brewster angle (15), as does the analytical reflection. And as the incidence tends to $90^{\circ}$, Luebbers reflection vanishes, i.e. the sheet is transparent to the incident wave, while Maloney reflection becomes total. All this clearly demonstrates that methods [3], [4], and [6], are erroneous at wide incidence angle, while the Maloney reflection is almost perfect at any incidence.

Concerning the transmission through the dielectric sheet, it can be computed from the reflection, since there is no loss in the sheet, so that $R^{2}+T^{2}=1$, as it can be easily verified numerically with (9).

Figs. 4-6 show results for a conducting sheet of thickness $d=\Delta x$ / $50=1 \mathrm{~mm}$. Fig. 4 is a comparison of FDTD theory with analytical reflection as a function of frequency, for incidence $\theta=80^{\circ}$ and conductivities $\sigma_{s}=0.1$ and $1 \mathrm{~S} / \mathrm{m}$. Above a certain frequency, that depends on the conductivity, Luebbers method deviates from the analytical solution, while Maloney method remains correct. Fig. 5 is a comparison as a function of the incidence angle, at 10 and $100 \mathrm{MHz}$ and conductivity $0.1 \mathrm{~S} / \mathrm{m}$. Up to $70^{\circ}$ the reflection is almost independent of frequency and both the Luebbers and Maloney methods agree with the analytical solution. At a higher incidence, the reflection depends on frequency and the Luebbers method becomes erroneous, as in the case of a dielectric. In particular, the Luebbers reflection vanishes while the Maloney reflection tends to unity, in accordance with the physical reflection. Notice the agreement of FDTD results computed up to $\theta=80^{\circ}$ (prohibitive CPU time for larger angles).

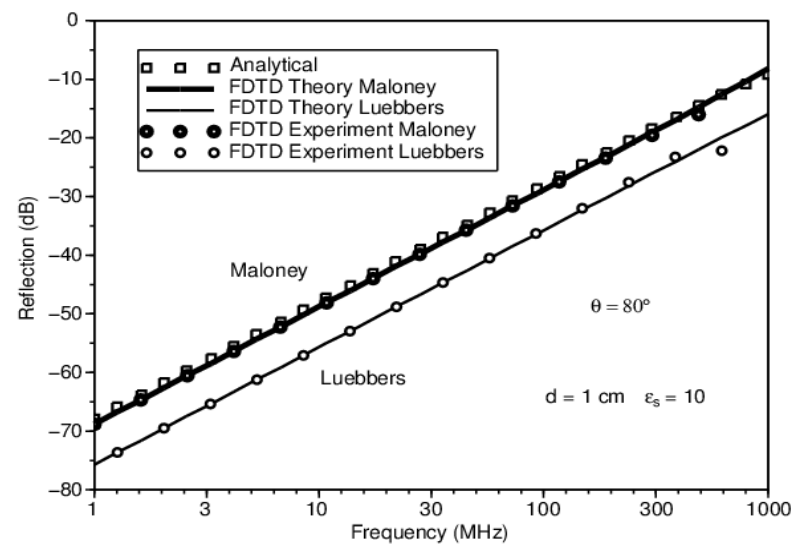

Fig. 2. Reflection at incidence angle $\theta=80^{\circ}$ from a dielectric sheet of $\varepsilon_{s}=10$ and thickness $d=\Delta x / 5=1 \mathrm{~cm}$. Comparison of analytical reflection (3) with Luebbers and Maloney reflections computed with FDTD theory (9), and with FDTD experiments.

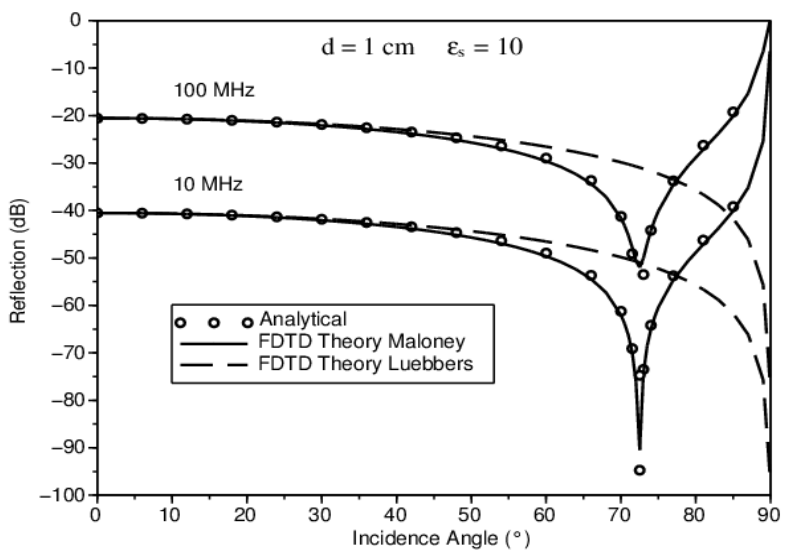

Fig. 3. Reflection from a dielectric sheet of $\varepsilon_{s}=10$ and thickness $d=\Delta x / 5=1$ $\mathrm{cm}$ at $10 \mathrm{MHz}$ and $100 \mathrm{MHz}$, as a function of incidence angle $\theta$.

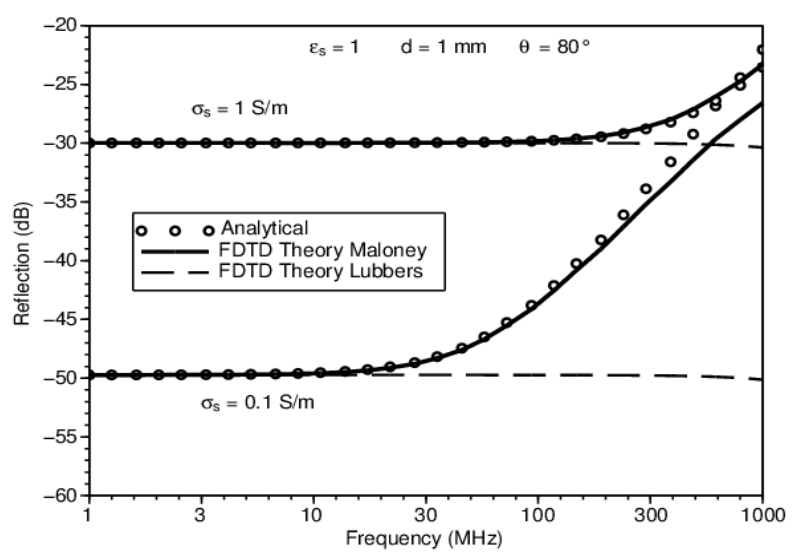

Fig. 4. Reflection at incidence angle $\theta=80^{\circ}$ from a conducting sheet of thickness $d=\Delta x / 50=1 \mathrm{~mm}$ and conductivities 0.1 and $1 \mathrm{~S} / \mathrm{m}$.

Fig. 6 depicts the transmission coefficient of the sheet, for three conductivities. Although it is plotted at $100 \mathrm{MHz}$, it is valid as well at any frequency since the transmission is almost independent of frequency. The Maloney and Luebbers methods appear as superimposed at any incidence angle in Fig. 6. In fact, at grazing 
incidence Maloney (9) and the exact (3) transmissions vanish, while Luebbers transmission tends to unity which means that the sheet is transparent to the wave. This is illustrated in Table I and can be verified with (9) where when $\alpha=1$ and $\theta$ tends to $90^{\circ}, w$ and $\psi_{u}$ vanish, $\chi$ tends to infinity, and thus $T$ in (9) tends to unity.

Reflection or transmission in other situations can be easily computed using (9). For instance, it can be shown that $R$ and $T$ are almost left unchanged when placing the plane where $E$ is split behind the sheet instead of in front of it. This just corresponds to permuting $\left(\varepsilon_{a}, \sigma_{a}\right)$ with $\left(\varepsilon b, \sigma_{b}\right)$ in $(9)$.

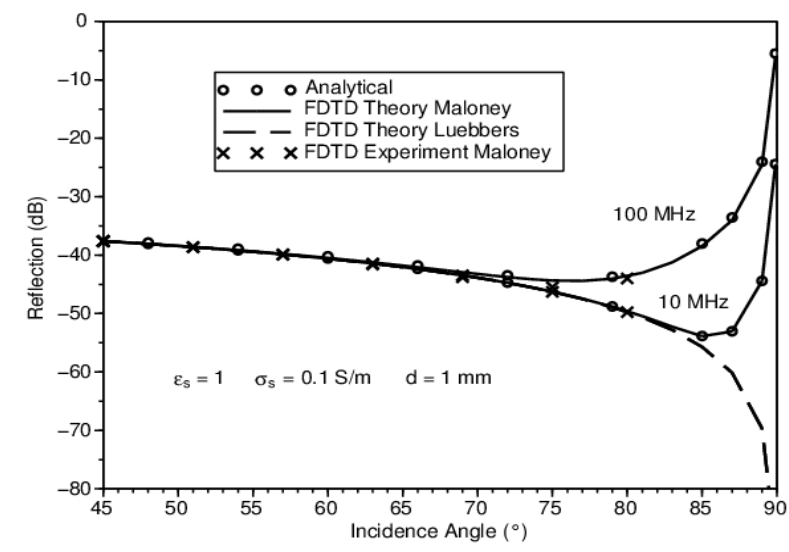

Fig. 5. Reflection from a conducting sheet of thickness $d=\Delta x / 50$ and conductivity $0.1 \mathrm{~S} / \mathrm{m}$, at frequencies 10 and $100 \mathrm{MHz}$.

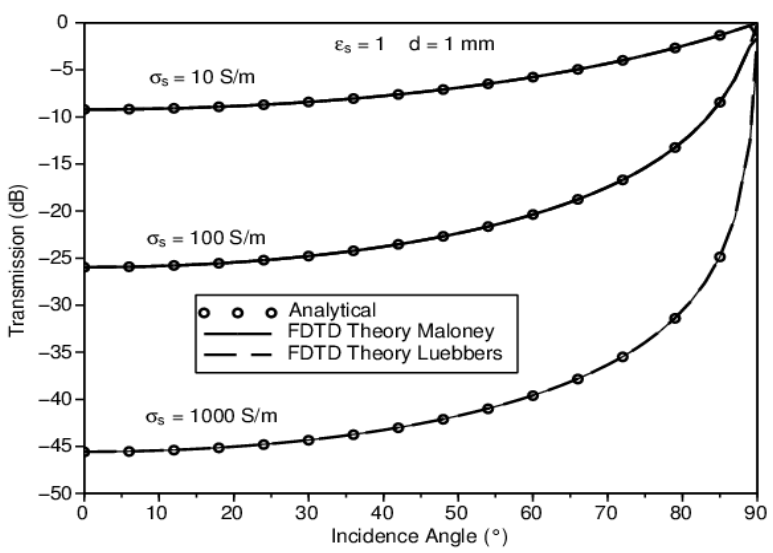

Fig. 6. Transmission of a sheet of thickness $d=\Delta x / 50=1 \mathrm{~mm}$ and conductivities 10, 100, $1000 \mathrm{~S} / \mathrm{m}$. Maloney and Luebbers curves are superimposed. Plotted at $100 \mathrm{MHz}$, the figure is valid at any frequency.

TABLE I

FDTD TRANSMISSION (dB) OF THE SHEET OF FIG. 6 AT WIDE INCIDENCE $\theta$, FOR $\sigma_{\mathrm{s}}=10 \mathrm{~S} / \mathrm{M}$ AND FREQUENCY $100 \mathrm{MHZ}$.

\begin{tabular}{|c|c|c|c|c|l|l|}
\hline$\theta\left(^{\circ}\right)$ & 85 & 89 & 89.9 & 89.99 & 89.999 & 89.9999 \\
\hline Luebbers & -1.32 & -0.281 & -0.029 & -0.003 & -0.000 & -0.000 \\
\hline Maloney & -1.32 & -0.298 & -1.371 & -15.6 & -35.0 & -50.7 \\
\hline
\end{tabular}

\section{Simplified Maloney Implementation for Dielectric Sheet}

In the case of a dielectric sheet, the two $E$ components perpendicular to the sheet, i.e. $E_{x_{-} \text {out }}$ and $E_{x_{-} i n}$ in Fig. 1, can be replaced with a unique component $E_{x_{-} \text {equ }}$ advanced using the standard FDTD equation with the fictitious relative permittivity

$$
\varepsilon_{\text {equ }}=1 /\left(\alpha+\beta / \varepsilon_{s}\right) .
$$

With the standard FDTD equation in place of (4b) and (4c), (8b) and (8c) are replaced with a unique equation

$$
T_{x_{-} \text {equ }} \sin (\omega \Delta t / 2)=-\frac{c \Delta t}{\varepsilon_{\text {equ }} \Delta y} T_{z} \sin \left(k_{y} \Delta y / 2\right)
$$

and $\left(\alpha T_{x_{\_} \text {in }}+\beta T_{x_{\_} \text {out }}\right)$ in (8d) is replaced with $T_{x_{\text {eequ }}}$ from (17). This yields the same equation as by substituting ( $8 \mathrm{~b})$ and ( $8 \mathrm{c}$ ) into (8d). The $R$ and $T$ solutions are thus the same as those of the initial set (8), rigorously. The implementation of a dielectric sheet is then slightly simpler than that with the split field [1] whilst the FDTD results are left unchanged.

\section{CONCLUSION}

In contrast to the simpler method [3], or similar methods [4] and [6], the Maloney and Smith method [1], that was also used in [11] with a non-orthogonal FDTD grid, provides us with accurate reflection and transmission when a plane wave strikes a dielectric or conducting thin sheet at any incidence angle. It thus appears as desirable to use method [1] in FDTD computer codes instead of other methods. However, the difference in the results may be either negligible or significant in practical applications, depending on the permittivity and conductivity of the sheet and on the incident wave. Further investigations will compare methods [1] and [3] in realistic applications and with canonical objects composed with several sheets of finite sizes. The comparisons will also be extended to the dispersive sheet method reported in [12].

\section{REFERENCES}

[1] G. Maloney, and G.S. Smith, "The efficient modeling of thin material sheets in the finite-difference time-domain (FDTD) method", IEEE Trans. Ant. Propag., vol. 40, pp. 323-330, 1992.

[2] P.A. Tirkas and K.R. Demarest, "Modeling of thin dielectric structures using the finite-difference time-domain technique", IEEE Trans. Ant. Propag., vol. 39, pp. 1338-1344, 1991.

[3] R.J. Luebbers and K. Kunz, "FDTD modeling of thin impedance sheets", IEEE Trans. Ant. Propag. Vol. 40, pp. 349-351, 1992.

[4] C.J. Railton and J.P. McGreehan, "An analysis of microstrip with rectangular and trapezoidal conductor cross sections", IEEE Trans. Micr. Th. Tech., vol. 38, pp. 1017-1022, 1990.

[5] L.-K. Wu and L.H-H. Han, "Implementation and application of resistive sheet boundary condition in the finite-difference time-domain method", IEEE Trans. Ant. Propag. vol. 40, pp. 628-633, 1992.

[6] J.-P. Bérenger, "Plaques minces aux différences finies", Colloque sur la Compatibilité Electromagnétique, Lyon, pp. 298-303, 1992.

[7] K. S. Yee, "Numerical solution of initial value boundary problems involving Maxwell's equations in isotropic media", IEEE Trans. Ant. Propag., voL. 14, pp. 302-307, 1966.

[8] A. Taflove and S. Hagness, "Computational Electrodynamics: The finitedifference time-domain method", Artech House, 2005.

[9] J. G. Maloney, and G.S. Smith, "A comparison of methods for modeling electrically thin dielectric and conducting sheets in the finite-difference time-domain (FDTD) method", IEEE Trans. Ant. Propag., vol. 41, pp. 690-694, 1993.

[10] J.-P. Bérenger, "A perfectly matched layer for the absorption of electromagnetic waves", J. Comput. Phys., vol. 114 pp. 185-200, 1994.

[11] E. A. Navarro, J. Segura, A. Soriano, and V. Such, "Modeling of thin curved sheets with the curvilinear FDTD", IEEE Trans. Ant. Propag., vol. 52, pp. 342-346, 2004.

[12] K. Tekbas, F. Costen, J.-P. Bérenger, R. Himeno, and H. Yokota, "Subcell modeling of frequency-dependent thin layers in the FDTD method", IEEE Trans. Ant. Propag., vol. 65, pp. 278-286, 2017. 
